\title{
Mathematical Modeling towards the Dynamical Interaction of Leptospirosis
}

\author{
Muhammad Altaf Khan ${ }^{1, *}$, Saeed Islam ${ }^{1}$ and Sher Afzal Khan ${ }^{2}$ \\ ${ }^{1}$ Department of Mathematics, Abdul Wali Khan University, Mardan, Khyber Pakhtunkhwa, Pakistan \\ ${ }^{2}$ Department of Computer Science, Abdul Wali Khan University, Mardan, Khyber Pakhtunkhwa, Pakistan
}

Received: 16 May. 2013, Revised: 20 Sep. 2013, Accepted: 21 Sep. 2013

Published online: 1 May. 2014

\begin{abstract}
In this work, we extend the mathematical model of leptospirosis disease by taking into account the exposed individuals, the related death rate and the transmission coefficients between susceptible human and infected vector. Initially, we present the local asymptotical stability of both the disease-free and endemic equilibrium. We use the Lyapunov function theory with some sufficient conditions. This shows the global stability of both the disease-free and endemic equilibrium. Further, we present the bifurcation of the model and exhibit that the local asymptotical stability of the disease-free and endemic equilibrium co-exists with the threshold quantity. Finally, we discuss the numerical results.
\end{abstract}

Keywords: Leptospirosis, mathematical models, Reproduction number, qualitative behavior, numerical simulations

\section{Introduction}

Mathematical formulation play an important role to present the transmission dynamics of different diseases. Among the diseases leptospirosis disease is one of the infectious disease which cause by a bacteria called leptospira. Human as well as Mammals are mostly infected from this disease. Leptospirosis is a zoonotic bacteriological disease, caused by members of the genus Leptospira. Due to the greater incidence in tropical regions, it is considered one of the most geographically widespread zoonosis in the world. Spectrum of human diseases caused by Leptospira broad, ranging from subclinical Infections to severe infections multiple organ dysfunction syndrome, sometimes fatal completion[1,2,3, $4,5]$.

Risk factor of the disease are, Rice planters, sewer cleaners, workers cleaning canals, agriculture labor easily contract this disease. In many model the exposed class define for different diseases [21], they consider the exposed class for human population, and use the transmission and carried out the dynamics for his model. Many models have been modeled to represent the compartmental dynamics of both the susceptible, infected and recovered human and vector population $[6,7,8]$. Pongsuumpun et al. [9] developed mathematical models to study the behavior of leptospirosis disease. In their work, they represent the rate of change for both vector and human population. The human population is further divided into two main groups Juveniles and adults. Triampo et al. [10] considered a deterministic models for the transmission of leptospirosis disease presented in [10]. In their work they considered a number of leptospirosis disease in Thailand and shown the numerical simulations. Zaman [11] considered the real data presented in [11] to study the dynamical behavior and role of optimal control theory of this disease, for more references [19, 18, 20,21,22].

In this paper, we extend the work of [11] by adding the exposed class $E_{h}$ to human population and the exposed class $E_{v}$ to the vector population, death rate to human population and vector population, a transmission coefficient between susceptible vector and infected human. First, we combine both the model to obtained a single model, then find the local asymptotical stability of the (DFE) and then find the local stability of endemic equilibrium and bifurcation of the model. Then we present, the bifurcation analysis and global asymptotical stability of the Disease-free and then find the endemic equilibrium by using the lyapunov function. For the local stability of the (DFE) and (EE) we introduce the basic

\footnotetext{
*Corresponding author e-mail: altafdir@gmail.com
} 
reproduction number. We also discuss the numerical result.

The paper is organized as follows. In Section 2 we present the mathematical formulation. Section 3 we show the local stability of both the disease-free and endemic equilibrium with bifurcation analysis of the model. Section 4 is devoted to the global stability of both the disease-free and endemic equilibrium. In Section 5, we presents numerical simulation of the model using the real data of Thailand.

\section{Model Formulation}

In this section, we combine the model presented in [11] we add the exposed class for both vector and human population. By the interaction of both non linear models of human and vector (rats) to a new single model of constructing system of seventh differential equations. To formulate our model, we assume that, $S_{h}(t)$ represent of susceptible human, $E_{h}(t)$ is the exposed class for human, $I_{h}(t)$ represent infected human, $R_{h}(t)$ represent the recovered class for human at time $t$. For the vector population, we assume that $S_{v}(t)$ represent the susceptible vector, $E_{v}(t)$ represent exposed class , $I_{v}(t)$, represent the class of infected vector at time $t$. Thus the total population of human is $N_{h}=S_{h}(t)+E_{h}(t)+I_{h}(t)+R_{h}(t)$, and vector population is $N_{v}=S_{v}(t)+E_{v}(t)+I_{v}(t)$. The interaction of both the model is presented in flow chart in Figure 1.

By the above mention modification, the system of nonlinear differential equation is given by

$$
\begin{aligned}
\frac{d S_{h}}{d t} & =a_{1}-\mu_{0} S_{h}-\beta_{1} S_{h} I_{v}-\alpha_{1} S_{h}+\lambda_{h} R_{h}, \\
\frac{d E_{h}}{d t} & =\beta_{1} S_{h} I_{v}+\alpha_{1} S_{h}-\mu_{0} E_{h}-\alpha_{h} E_{h}, \\
\frac{d I_{h}}{d t} & =\alpha_{h} E_{h}-\mu_{0} I_{h}-\mu_{h} I_{h}-\delta_{h} I_{h}, \\
\frac{d R_{h}}{d t} & =\delta_{h} I_{h}-\mu_{0} R_{h}-\lambda_{h} R_{h}, \\
\frac{d S_{v}}{d t} & =a_{2}-\delta_{0} S_{v}-\beta_{2} S_{v} I_{h}, \\
\frac{d E_{v}}{d t} & =\beta_{2} S_{v} I_{h}-\delta_{0} E_{v}-\alpha_{v} E_{v} \\
\frac{d I_{v}}{d t} & =\alpha_{v} E_{v}-\delta_{0} I_{v}-\delta_{v} I_{v}
\end{aligned}
$$

with initial conditions

$$
S_{h} \geq 0, E_{h} \geq 0, I_{h} \geq 0, R_{h} \geq 0, S_{v} \geq 0, E_{v} \geq 0, I_{v} \geq 0 .
$$

Here $a_{1}$ is the recruitment rate for human, $\beta_{1}$ is the transmission coefficient between human and infected vector. The transmission rate between $S_{v}$ and $I_{h}$ is shown by $\beta_{2}$, the susceptible human infected at rate of $\alpha_{1}$. The natural death rate for the human population is $\mu_{0}$, while the infected human dies from the disease at the rate of $\mu_{h}$. The growth rate for the vector population is denoted by

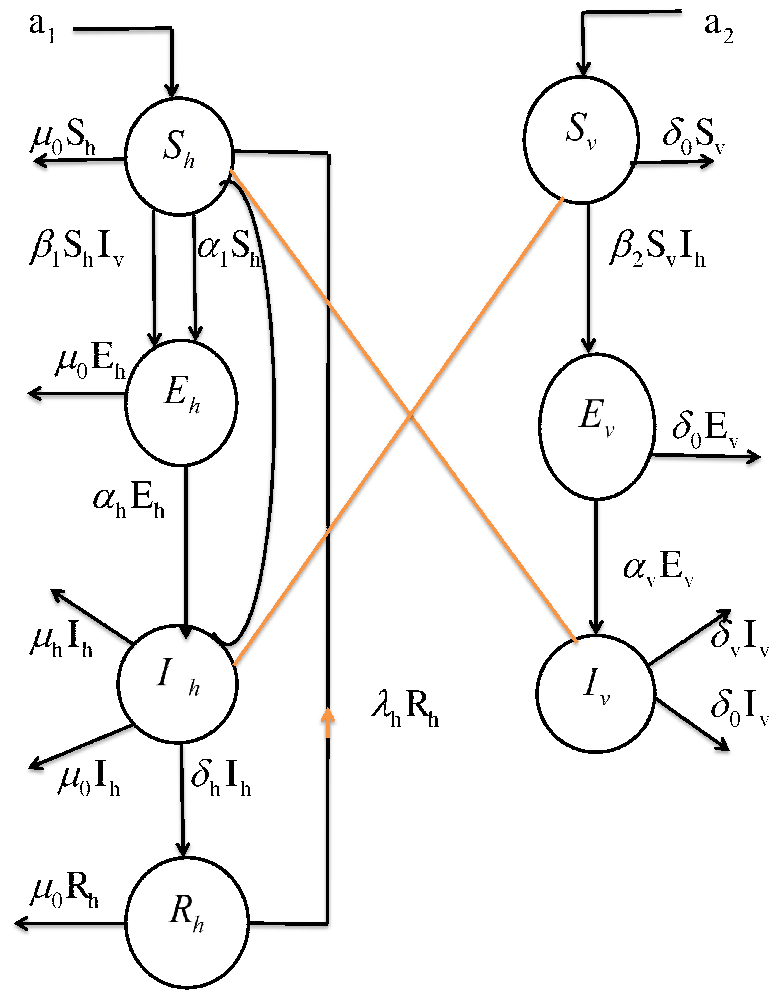

Fig. 1: The plot represents the flow diagram of the interaction of human and vector.

$a_{2}$, the natural death rate for the vector population is $\delta_{0}$, at $\alpha_{h}$ rate the exposed human move to infected class of human, while at the rate of $\alpha_{v}$ the exposed vector move to the infected vector class. The infected vector dies at the rate of $\delta_{v}$.

We obtain the total dynamics of human population by adding the human Subclasses is given by,

$$
\frac{d N_{h}}{d t}=a_{1}-\mu_{0} N_{h}-\mu_{h} I_{h} .
$$

Similarly adding the vector Subclasses we get the total dynamics of vector population is given by

$$
\frac{d N_{v}}{d t}=a_{2}-\delta_{0} N_{v}-\delta_{v} I_{v}
$$

From (1) and (2), we get,

$$
\frac{d N_{h}}{d t} \leq a_{1}-\mu_{0} N_{h} \quad \text { and } \quad \frac{d N_{v}}{d t} \leq a_{2}-\delta_{0} N_{v} .
$$

Now, we can prove that

$$
\frac{d N_{h}}{d t} \leq a_{1}-\mu_{0} N_{h} \leq 0 \quad \text { for } \quad N_{h}>\frac{a_{1}}{\mu_{0}},
$$




$$
\frac{d N_{v}}{d t} \leq a_{2}-\delta_{0} N_{v} \leq 0 \quad \text { for } \quad N_{v}>\frac{a_{2}}{\delta_{0}}
$$

For the system (1) the feasible region is

$\Omega=\left[\left(S_{h}(t), E_{h}(t), I_{h}(t), R_{h}(t), S_{v}(t), E_{v}(t), I_{v}(t)\right) \quad \in\right.$ $\left.R_{+}^{7}, \quad\left(N_{h} \leq \frac{a_{1}}{\left(\mu_{0}+\alpha_{1}\right)}, \quad N_{v} \leq \frac{a_{2}}{\delta_{0}}\right)\right]$

Proposition Let the variables $\left(S_{h}(t)+E_{h}(t)+I_{h}(t)+R_{h}(t)\right.$ of human population and the variables $\left.S_{v}(t)+E_{v}(t)+I_{v}(t)\right)$ of vector population is the solution of the system (1) with the associated initials conditions (2) and the set $\Omega$. Then $\Omega$ under system (1) is positively invariant and attracting.

Proof:To prove this we consider the Lyapunov function

$$
\begin{aligned}
N(t)= & \left(N_{h}(t)+N_{v}(t)\right)=\left(S_{h}(t)+E_{h}(t)+I_{h}(t)+R_{h}(t),\right. \\
& \left.S_{v}(t)+E_{v}(t)+I_{v}(t)\right) .
\end{aligned}
$$

Taking its time derivative. We get

$$
\frac{d N}{d t}=\left(a_{1}-\mu_{0} N_{h}-\mu_{h} I_{h}, a_{2}-\delta_{0} N_{v}-\delta_{v} I_{v}\right)
$$

We can easily prove that.

$$
\begin{aligned}
& \frac{d N_{h}}{d t} \leq a_{1}-\mu_{0} N_{h} \leq 0, \quad \text { for } \quad N_{h} \geq \frac{a_{1}}{\mu_{0}+\alpha_{1}}, \\
& \text { and } \frac{d N_{v}}{d t} \leq a_{2}-\delta_{0} N_{v} \leq 0, \quad \text { for } \quad N_{v} \geq \frac{a_{2}}{\delta_{0}}
\end{aligned}
$$

Its clear from (8) that $\frac{d\left(N_{h}, N_{v}\right)}{d t} \leq 0$. Here using comparison theorem [14] to show that $0 \leq\left(N_{h}, N_{v}\right) \leq\left(N_{h}(0) e^{-\mu_{0} t}+\right.$ $\left.\frac{a_{1}}{\mu_{0}+\alpha_{1}}\left(1-e^{-\mu_{0} t}\right), \quad N_{v}(0) e^{-\delta \delta_{0} t}+\frac{a_{2}}{\delta_{0}}\left(1-e^{-\delta_{0} t}\right)\right)$
$\stackrel{0}{\longrightarrow}$ we get,

$0 \leq\left(N_{h}, N_{v}\right) \leq\left(\frac{a_{1}}{\mu_{0}+\alpha_{1}}, \frac{a_{2}}{\delta_{0}}\right)$ and we conclude that $\Omega$ is an attracting set.

\section{Local Stability Analysis}

In this section, we find the disease free equilibrium for the system (1). Find the basic reproduction number $R_{o}$ called the threshold qunatity by the method developed by [17]. We introduce $R_{o}$ in DFE and also in the EE of the system (1) for the local stability. We show that the reproduction number $R_{0}$ co-exists with the disease-free and endemic equilibrium. To obtain the disease free equilibrium by setting left hand side of the system (1) equal to zero, around the point $E_{1}=\left(S_{h}^{0}, 0,0,0, S_{v}^{0}, 0,0\right)$. Solution of the system (1) yields, we obtained the Disease-free equilibrium around $E_{1}=\left(S_{h}^{0}, 0,0,0, S_{v}^{0}, 0,0\right)$ is, where

$$
S_{h}^{0}=\frac{a_{1}}{\left(\mu_{0}+\alpha_{1}\right)} \quad \text { and } \quad S_{v}^{0}=\frac{a_{2}}{\delta_{0}} .
$$

The quantity which described the disease by the quantity, which is called the threshold quantity ,

$$
R_{0}=\frac{a_{1} \alpha_{h} \alpha_{1} T_{4} T_{5} \delta_{0}+\alpha_{\nu} \alpha_{h} \beta_{1} \beta_{2} a_{2} a_{1}}{T_{1} T_{2} T_{4} T_{5}\left(\mu_{0}+\alpha_{1}\right) \delta_{0}},
$$

where

$$
\begin{gathered}
T_{1}=\left(\mu_{0}+\alpha_{h}\right), \quad T_{2}=\left(\mu_{0}+\mu_{h}+\delta_{h}\right), \quad T_{3}=\left(\mu_{0}+\lambda_{h}\right), \\
T_{4}=\left(\delta_{0}+\alpha_{v}\right), T_{5}=\left(\delta_{0}+\delta_{v}\right) .
\end{gathered}
$$

In the following we find the disease free state of the system (1) around $E_{1}$.

Theorem: The DFE about $E_{1}$ of the system (1) for $R_{0} \leq 1$, stable locally asymptotically, if $\delta_{0}>\frac{\left(\mu_{0}+\alpha_{1}\right)}{\mu_{0} T_{4} T_{5}}$ and $\left(\mu_{0}+\alpha_{1}\right)>\frac{\delta_{h} \alpha_{h} \alpha_{1} \lambda_{h}}{T_{1} T_{2} T_{3}}$, otherwise unstable.

Proof: To show that the system (1) is stable locally asymptotically setting left side of the system (1) equal zero get the following Jacobian matrix $J_{0}$ around $E_{1}$. See detail in Appendix 1.

\subsection{Endemic Equilibria and Bifurcation of the Model}

For the endemic equilibria of the system (1), we use $E_{2}=\left(S_{h}^{*}, E_{h}^{*}, I_{h}^{*}, R_{h}^{*}, S_{v}^{*}, E_{v}^{*}, I_{v}^{*}\right)$ and setting left hand side of the system (1) equal to zero, to get the equilibria.

$$
\begin{aligned}
S_{h}^{*} & =\frac{T_{1} T_{2} T_{4} T_{5}\left(\delta_{0}+\beta_{2} I_{h}^{*}\right) I_{h}^{*}}{\left(\alpha_{1} T_{4} T_{5} \alpha_{h}\left(\delta_{0}+\beta_{2} I_{h}^{*}\right)+\alpha_{h} \beta_{1} \alpha_{v} \beta_{2} a_{2} I_{h}^{*}\right)}, \\
E_{h}^{*} & =\frac{T_{2} I_{h}^{*}}{\alpha_{h}}, \\
R_{h}^{*} & =\frac{\delta_{h} I_{h}^{*}}{T_{3}}, \\
S_{v}^{*} & =\frac{a_{2}}{\delta_{0}+\beta_{2} I_{h}^{*}}, \\
E_{v}^{*} & =\frac{\beta_{2} a_{2} I_{h}^{*}}{T_{4}\left(\delta_{0}+\beta_{2} I_{h}^{*}\right)}, \\
I_{v}^{*} & =\frac{\alpha_{v} \beta_{2} a_{2} I_{h}^{*}}{T_{4} T_{5}\left(\delta_{0}+\beta_{2} I_{h}^{*}\right)} .
\end{aligned}
$$

Theorem For $R_{0} \geq 1$, the EEE around $E_{2}$ of the system (1) is locally asymptotically stable if the following inequalities are satisfied.

$\mu_{0}>\frac{\beta_{1} a_{2}}{T_{3} T_{4} T_{5}}$ and

$a_{2} \delta_{0}>\frac{\delta_{v} \beta_{2} \alpha_{h} \mu_{0}}{T_{1} T_{2} T_{3}\left(\mu_{0}+\alpha_{1}\right)}$,

otherwise unstable.

Proof: To prove the above theorem, setting left side of the system (1) equal to zero, around an endemic equilibrium $E_{2}$ give the Jacobian matrix,

See Appendix 2 in detail.

\subsection{Bifurcation of the Model}

To find the backward bifurcation of the system (1), and for the backward bifurcation one of the infected 
component at least non-zero. In the system (1) using the first equation and substituting the value of $S_{h}^{*}, I_{v}^{*}$ and $R_{h}^{*}$ and after the calculations we obtained for

$$
f\left(I_{h}^{*}\right)=A I_{h}^{* 2}+B I_{h}^{*}+C=0
$$

where,

$$
\begin{aligned}
\mathbf{A}= & a_{1} \beta_{2}^{2} T_{3}^{2} T_{4}^{2} T_{5}^{2}+a_{1} \beta_{2}^{2} T_{3} \alpha_{1} T_{4}^{2} T_{5}^{2}+\lambda_{h} \delta_{h} T_{3} \mu_{0} T_{4} T_{5} \beta_{2} \\
& +\lambda_{h} \delta_{h} T_{3} \beta_{1} \alpha_{v} \beta_{2} a_{2}+\lambda_{h} \delta_{h} \alpha_{1} T_{4} T_{5} \beta_{2}, \\
\mathbf{B}= & a_{1} T_{4} T_{5}\left[\delta_{0} T_{3}^{2} T_{4} T_{5} \beta_{2}+\delta_{0} T_{3}^{2} \beta_{1} \alpha_{v} \beta_{2} a_{2}+\delta_{0} T_{3}^{2} \alpha_{1} T_{4} T_{5} \beta_{2}\right. \\
& +\delta_{0} T_{3}^{2} T_{4} T_{5} \beta_{2} \delta_{0} T_{3}^{2} T_{4} T_{5} \beta_{2} \delta_{0} T_{3}^{2} \beta_{1} \alpha_{v} \beta_{2} a_{2} \\
& +\delta_{0} T_{3}^{2} \alpha_{1} T_{4} T_{5} \beta_{2}+\beta_{2} T_{3}^{2} T_{4} T_{5} \delta_{0}+\beta_{2}^{3} \beta_{1} \alpha_{v} a_{2} T_{3}^{2} \\
& +\beta_{2} T_{3}^{2} \alpha_{1} T_{4} T_{5} \delta_{v}-T_{3} \mu_{0} T_{4} T_{5} \beta_{2}+\beta_{1} \alpha_{v} \beta_{2} a_{2} T_{3} \\
& \left.+\alpha_{1} T_{4} T_{5} T_{3} \beta_{2}+\lambda_{h} \delta_{h} T_{3} \mu_{o} T_{4} T_{5} \delta_{0}+\lambda_{h} \delta_{h} \alpha_{1} T_{4} T_{5} \delta_{v}\right], \\
\mathbf{C}= & a_{1} T_{4}^{2} T_{5}^{2} \alpha_{1} \delta_{0} T_{3}\left(1-R_{0}\right) .
\end{aligned}
$$

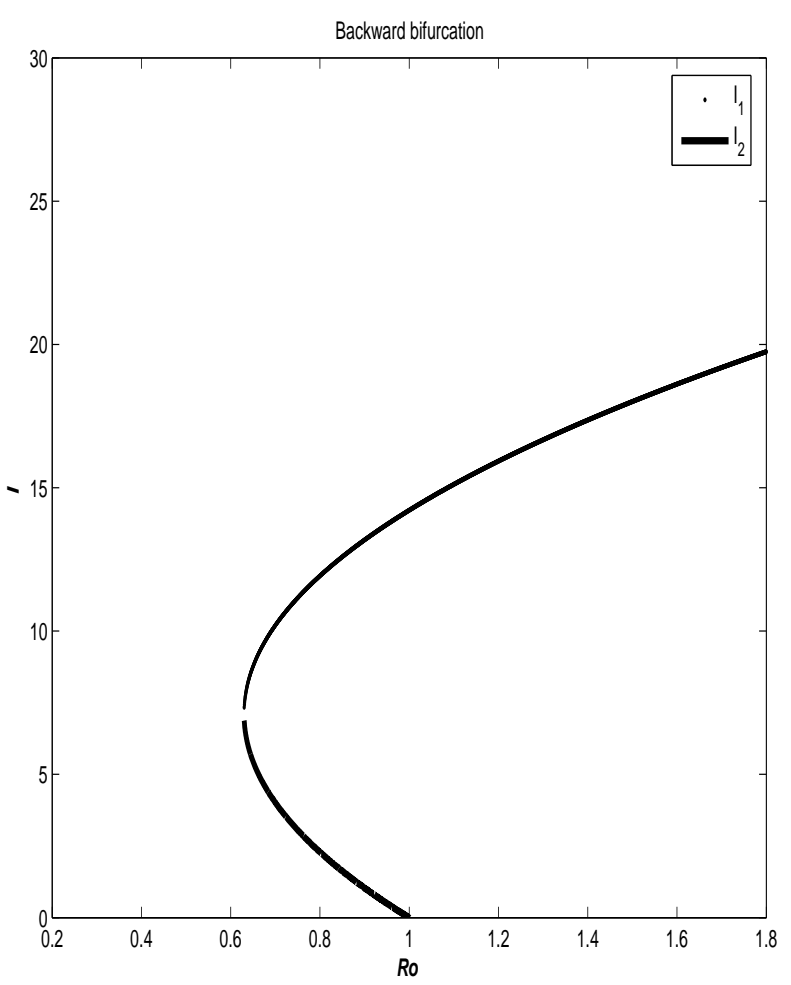

Fig. 2: The plot represents the bifurcation of the model.

The coefficient $A$ is positive always and $C$ depends upon
$R_{0}$, if $R_{0}<1$ then $C$ is positive and if $R_{0}>1$ then $C$ is negative. For $A>0$ the positive solution depends upon the sign of $C$ and $B$. For $R_{0}>1$ the equation(9) having two roots, positive and negative. If $R_{0}=1$, then we see that $C=0$ and we obtain a non-zero solution of equation (9), that is $-\frac{B}{A}$, is positive $\Longleftrightarrow B<0$, for $B<0$ then there exists a positive endemic equilibrium for $R_{0}=1$. It means that equilibria continuously depending upon $R_{0}$, this show that there exists an interval for $R_{0}$ which have two positive equilibria

$$
I_{0}=\frac{-B-\sqrt{B^{2}-4 A C}}{2 A}, \quad I_{1}=\frac{-B+\sqrt{B^{2}-4 A C}}{2 A} .
$$

The local stability of DFE co-exists with the local asymptotic stability of the EE when $R_{0}<1$, see $[15,16]$. For the backward bifurcation setting the discriminant $B^{2}-4 A C=0$ and then solving for the critical points of $R_{0}$ which is given by $R_{c}=1-\frac{B^{2}}{4 A a_{1} T_{4}^{2} T_{5}^{2} T_{3} \alpha_{1} \delta_{0}}$. If $R_{c}<R_{0}$ equivalently $B^{2}-A C>0$ and backward bifurcation occur for the points of $R_{0}$ such that $R_{c}<R_{0}<1$. To illustrate we consider represent in Figure 2: $a_{1}=23, a_{2}=12$, $\mu_{0}=0.0071, \lambda_{h}=0.066, \delta_{0}=0.0023, \delta_{v}=0.0029$, $\alpha_{v}=0.0081, \alpha_{1}=0.0001, \beta_{1}=0.0074, \beta_{2}=0.0073$, $\mu_{h}=0.0002$

\section{Global Stability (GS) Analysis of the Model}

In this section, we shows the global stability of the system (1) by using the lyapunov function of the disease-free state and then using the endemic equilibrium and find the GS of the endemic equilibrium. First we show the global stability in the following by defining the lyapunov function.

Theorem: The DFE around $E_{1}$ of the system (1) is GAS for $R_{0} \geq 1$, if $S_{v}=S_{v}^{0}, S_{h}=S_{h}^{0}$ and $\delta_{v} \geq \frac{T_{3}\left(\mu_{0}+\alpha_{1}\right)}{T_{4} T_{5} \alpha_{1}}$, otherwise unstable.

Proof: Here we show the GS of the disease-free state for the system (1) by using the lyapunov function.

$V(t)=W_{1} S_{h}+W_{2} E_{h}+W_{3} I_{h}+W_{4} R_{h}+W_{5} S_{v}$

$$
+W_{6} E_{v}+W_{7} I_{v}
$$

where $W_{i}(i=1 \ldots 7)$ are positive constants to be choosing later.

Taking derivative w.r to time $t$ of the above defined function, we have

$$
\begin{aligned}
V^{\prime}(t)= & W_{1} \frac{d S_{h}}{d t}+W_{2} \frac{d E_{h}}{d t} \\
& +W_{3} \frac{d I_{h}}{d t}+W_{4} \frac{d R_{h}}{d t}+W_{5} \frac{d S_{v}}{d t} \\
& +W_{6} \frac{d E_{v}}{d t}+W_{7} \frac{d I_{v}}{d t} .
\end{aligned}
$$

Using the system (1) we get,

$V^{\prime}(t)=W_{1}\left[a_{1}-\mu_{0} S_{h}-\beta_{1} S_{h} I_{v}-\alpha_{1} S_{h}+\lambda_{h} R_{h}\right]$ 


$$
\begin{aligned}
& +W_{2}\left[\beta_{1} S_{h} I_{v}+\alpha_{1} S_{h}-T_{1} E_{h}\right]+W_{3}\left[\alpha_{h} E_{h}-T_{2} I_{h}\right] \\
& +W_{4}\left[\delta_{h} I_{h}-T_{3} R_{h}\right]+W_{5}\left[a_{2}-\delta_{0} S_{v}-\beta_{2} S_{v} I_{h}\right]+ \\
& W_{6}\left[\beta_{2} S_{v} I_{h}-T_{4} E_{v}\right]+W_{7}\left[\alpha_{v} E_{v}-T_{5} I_{v}\right] .
\end{aligned}
$$

Where (I) denotes the derivative w.r.t time, and after the simplifications we get

$$
\begin{aligned}
V^{\prime}(t)= & E_{v}\left[W_{7} \alpha_{v}-W_{6} T_{4}\right]+\beta_{2} S_{v} I_{h}\left[W_{6}-W_{5}\right] \\
& +E_{h}\left[W_{3} \alpha_{h}-W_{2} T_{1}\right]+\alpha_{1} S_{h}\left[W_{2}-W_{1}\right] \\
& +\beta_{1} S_{h} I_{v}\left[W_{2}-W_{1}\right]+R_{h}\left[W_{4} T_{3}-W_{1} \lambda_{h}\right] \\
& +W_{1} a_{1}-W_{1} \mu_{0} S_{h}-W_{3} T_{2} I_{h} \\
& +W_{4} \delta_{h} I_{h}-W_{7} T_{5} I_{v}+W_{5} a_{2}-W_{5} \delta_{0} S_{v} .
\end{aligned}
$$

Now we choosing the constants,

$W_{1}=W_{2}=\alpha_{h}, W_{3}=T_{1}, W_{4}=\frac{\alpha_{h} \lambda_{h}}{T_{3}}, W_{5}=\alpha_{v}, W_{6}=\alpha_{v}$, $W_{7}=T_{4}$ and $a_{1}=\left(\mu_{0}+\alpha_{1}\right) S_{h}^{0}, \quad a_{2}=\delta_{0} S_{v}^{0}, \quad \mu_{0}=\left(\mu_{0}+\right.$ $\left.\alpha_{1}\right)$,

to obtain, the following equation after some arrangements.

$$
\begin{aligned}
V^{\prime}(t)= & -\mu_{0} T_{4} T_{5} \delta_{0} T_{3} \alpha_{h}\left(\mu_{0}+\alpha_{1}\right)\left[S_{h}-S_{h}^{0}\right] \\
& -\mu_{0} T_{4} T_{5} \delta_{0} \alpha_{v} T_{3} \delta_{0}\left[S_{v}-S_{v}^{0}\right]-\mu_{0} T_{4}^{2} T_{5}^{2} \delta_{0} T_{3} I_{v} \\
& -I_{h}\left[T_{1} T_{2} T_{3}\left(\mu_{0}+\alpha_{1}\right)\left(R_{0}-1\right)-\alpha_{h} \lambda_{h} \delta_{h} \mu_{o} T_{4} T_{5} \delta_{0}\right. \\
& \left.-T_{1} T_{2}\left(T_{4} T_{5} \alpha_{1} \delta_{v}-T_{3}\left(\mu_{0}+\alpha_{1}\right)\right)\right] .
\end{aligned}
$$

$V^{\prime}(t)$ is negative for $R_{0} \geq 1$ and $\delta_{v} \geq \frac{T_{3}\left(\mu_{0}+\alpha_{1}\right)}{T_{4} T_{5} \alpha_{1}}$.

Also $\quad V^{\prime}(t)=0 \quad$ is r zero for $S_{h}=S_{h}^{0}, S_{v}=S_{v}^{0}, R_{h}=E_{h}=I_{h}=I_{v}=E_{v}=0$.

Hence by Lassalle's principle [13] $E_{1}$ is globally asymptotically stable. This end the proof.

Next, we show that the endemic equilibrium point $E_{2}$ of the system (1) is globally asymptotically stable. In order to do this, we define the Lyapunov function and show that the endemic equilibrium point $E_{2}$ of the system (1) is globally asymptotically stable.

Theorem: The endemic equilibrium $E_{2}$ of the system (1) is stable globally asymptotically, if $S_{v}^{*}=1$, otherwise unstable.

Proof: To show that the endemic equilibrium is globally asymptotically stable, we define the following Lyapunov function.

$$
\begin{aligned}
L(t) a= & W_{1}\left(S_{h}-S_{h}^{*}-\frac{S_{h}}{S_{h}^{*}}+1\right)+W_{2}\left(S_{v}-S_{v}^{*}-\frac{S_{v}}{S_{v}^{*}}+1\right) \\
& +W_{3} E_{h}+W_{4} I_{h}+W_{5} R_{h}+W_{6} E_{v}+W_{7} I_{v} .
\end{aligned}
$$

where $W_{i}(i=1 \ldots 7)$ are positive constants will chosen later.

Taking the time derivative of the above function along the solution of system (1) we get,

$$
\begin{aligned}
L^{\prime}(t)= & W_{1}\left(\frac{S_{h}^{*}-1}{S_{h}^{*}}\right)\left[a_{1}-\mu_{0} S_{h}-\beta_{1} S_{h} I_{v}-\alpha_{1} S_{h}+\lambda_{h} R_{h}\right] \\
& +W_{2}\left(\frac{S_{v}^{*}-1}{S_{v}^{*}}\right)\left[a_{2}-\delta_{0} S_{v}-\beta_{2} S_{v} I_{h}\right]+W_{3}\left[\beta_{1} S_{h} I_{v}+\alpha_{1} S_{h}-T_{1} E_{h}\right] \\
& +W_{4}\left[\alpha_{h} E_{h}-T_{2} I_{h}\right]+W_{5}\left[\delta_{h} I_{h}-T_{3} R_{h}\right]+W_{6}\left[\beta_{2} S_{h} I_{v}-T_{4} E_{v}\right] \\
& +W_{7}\left[\alpha_{v} E_{v}-T_{5} I_{v}\right],
\end{aligned}
$$

where ( 1 ) denotes time derivatives . After a little arrangement we get,

$$
\begin{aligned}
L^{\prime}(t)= & a_{1} W_{1}\left(\frac{S_{h}^{*}-1}{S_{h}^{*}}\right)-\mu_{0} \frac{S_{h}}{S_{h}^{*}} W_{1}\left(S_{h}^{*}-1\right)-\beta_{2} W_{1} \frac{S_{h}}{S_{h}^{*}}\left(S_{h}^{*}-1\right) I_{v} \\
& +\lambda_{h} W_{1}\left(\frac{S_{h}^{*}-1}{S_{h}^{*}}\right) R_{h}+W_{2} a_{2}\left(\frac{S_{v}^{*}-1}{S_{v}^{*}}\right)-W_{2} \delta_{0} \frac{S_{v}}{S_{v}^{*}}\left(S_{v}^{*}-1\right)- \\
& W_{5} T_{3} R_{h}-W_{7} T_{5} I_{v}+\left[W_{7} \alpha_{v}-W_{6} T_{4}\right] E_{v}+\left[W_{5} \delta_{h}-W_{4} T_{2}\right] I_{h} \\
& +\left[W_{4} \alpha_{h}-W_{3} T_{1}\right] E_{h}+\left[W_{6} \beta_{2}-\beta_{1} W_{1}\left(\frac{S_{h}^{*}-1}{S_{h}^{*}}\right)\right] I_{v} \\
& +\left[W_{6}-W_{2}\left(\frac{S_{v}^{*}-1}{S_{v}^{*}}\right)\right] \beta_{2} S_{v} I_{h} .
\end{aligned}
$$

Choosing the constants, $W_{1}=\frac{\left(S_{v}^{*}-1\right) \beta_{2} S_{h}^{*}}{S_{v}^{*}\left(S_{h}^{*}-1\right) \beta_{1}} \quad W_{2}=1$, $W_{3}=\frac{\left(S_{v}^{*}-1\right) \beta_{2} T_{1}}{S_{v}^{*} \alpha_{h}}, \quad W_{4}=\frac{\left(S_{v}^{*}-1\right) \beta_{2} T_{1}}{S_{v}^{*} \alpha_{h}^{2}}$, $W_{5}=\frac{\left(S_{v}^{*}-1\right) \beta_{2} T_{1} T_{2}}{\alpha_{h}^{2} S_{v}^{*} \delta_{h}}, \quad W_{6}=\frac{\left(S_{v}^{*}-1\right)}{S_{v}^{*}}, \quad W_{7}=\frac{\left(S_{v}^{*}-1\right) T_{4}}{S_{v}^{*} \alpha_{v}}$, $a_{1}=\left(\mu_{0}+\alpha_{1}\right) S_{h}^{*}, \quad a_{2}=\delta_{0} S_{v}^{*}$. after the some simplifications we obtain,

$$
\begin{aligned}
L^{\prime}(t)= & \frac{\left(\mu_{0}+\alpha_{1}\right) S_{h}^{*}\left(S_{v}^{*}-1\right) \beta_{2}}{\beta_{1} S_{v}^{*}}-\frac{\mu_{0} S_{h}\left(S_{v}^{*}-1\right) \beta_{2}}{\beta_{1} S_{v}^{*}}-\frac{S_{h}\left(S_{v}^{*}-1\right) \beta_{2} I_{v}}{S_{v}^{*}} \\
& +\frac{\lambda_{h}\left(S_{v}^{*}-1\right) \beta_{2} R_{h}}{S_{v}^{*} \beta_{1}}+\delta_{0}\left(S_{v}^{*}-1\right)-\frac{\delta_{0} S_{v}\left(S_{v}^{*}-1\right)}{S_{v}^{*}} \\
& +\frac{S_{h}^{2}\left(S_{v}^{*}-1\right) \beta_{2} \beta_{1} T_{1} I_{v}}{S_{v}^{*} \alpha_{h}}-\frac{\left(S_{v}^{*}-1\right) \beta_{2}^{2} T_{1} T_{2} T_{3} R_{h}}{\alpha_{h}^{2} S_{v}^{*} \delta_{h}}-\frac{\left(S_{v}^{*}-1\right) T_{4}}{S_{v}^{*} \alpha_{v}} .
\end{aligned}
$$

$L^{\prime}(t)$ is negative for $S_{v}^{*}=1$ and $L(t)$ is zero for all $S_{h}=S_{h}^{*}, S_{v}=S_{v}^{*}, R_{h}=0, E_{h}=0, I_{h}=0, E_{v}=0, I_{v}=0$.

Hence by the theorem of asymptotic stability [13], the endemic equilibrium state $E_{2}$ is globally asymptotically stable. This completes the proof.

\section{Numerical Simulation and Discussion}

In this section, we present the numerical simulation of the proposed model (1). The given system (1) is solve numerically by using the well-known method, called Runge-Kutta order four scheme by using Matlab.

Figure 3 represents the dynamical behavior of human population. The bold line represent the population of susceptible individual. The exposed individuals are represented by the dashed line. The dotted line show the the population of infected individuals. The dashed dotted line representing the recovered human population from the infection. The parameter values that we used in the numerical simulations are $a_{1}=5 \times 10^{-2}$, $\beta_{1}=0.04, \alpha_{1}=0.06, \beta_{2}=0.0078, \lambda_{h}=2.85 \times 10^{-3}$, 


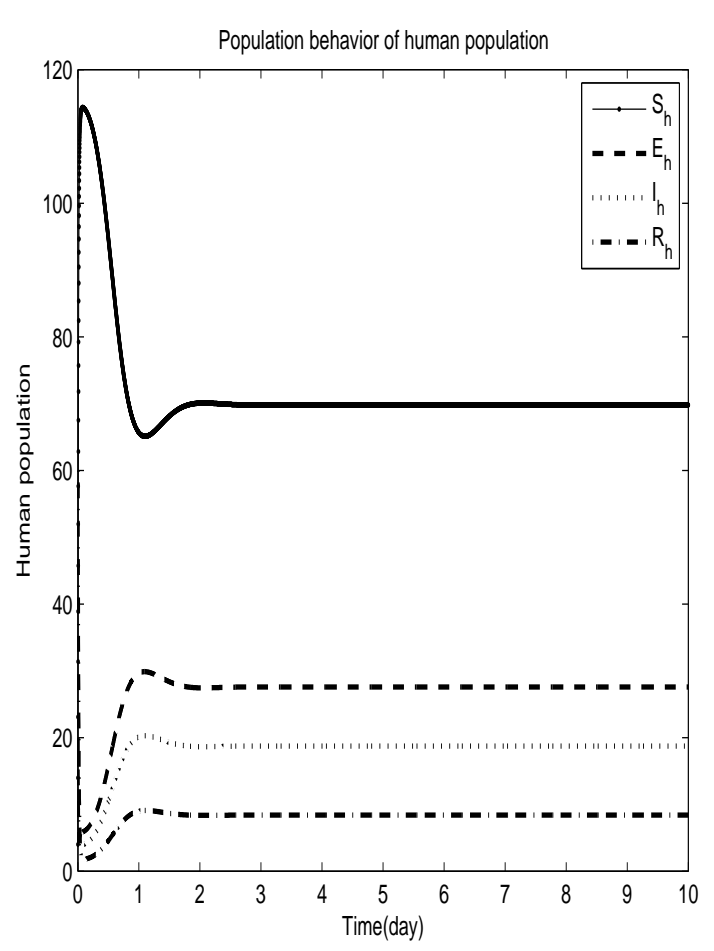

Fig. 3: The plot represents the human population.

$\mu_{o}=9 \times 10^{-4}, \alpha_{h}=2 \times 10^{-6}$, $\mu_{h}=8 \times 10^{-4}, \delta_{h}=2.1 \times 10^{-3}, a_{2}=2, \delta_{o}=1 \times 10^{-1}$, $\alpha_{v}=1 \times 10^{-2}, \delta_{v}=10^{-3}$.

Figure 4 represent the dynamical behavior of the vector population. The bold line show the class of susceptible vector. The dashed line indicate the exposed class of vector population and the dotted line represent the population of infected vector.

\section{Conclusion}

In this paper, we modify the model by the classes of exposed to the human and vector population, Disease mortality in humans and vector population in the infected class vector transmission coefficients between human exposure and infection.

We supply local asymptotic stability of the DFE and EE. For the endemic equilibrium $R_{0} \geq 1$ is asymptotically stable and $R_{0}<1$ the DFE locally asymptotically stable. We also show backward bifurcation for the system (1). With Lyapunov's theory of functions global stability of the equilibrium state, free from disease and endemic is obtained. We make sure that these new assumptions and analysis of an appropriate biological point of the previous assumptions, without exposed Class, disease mortality in

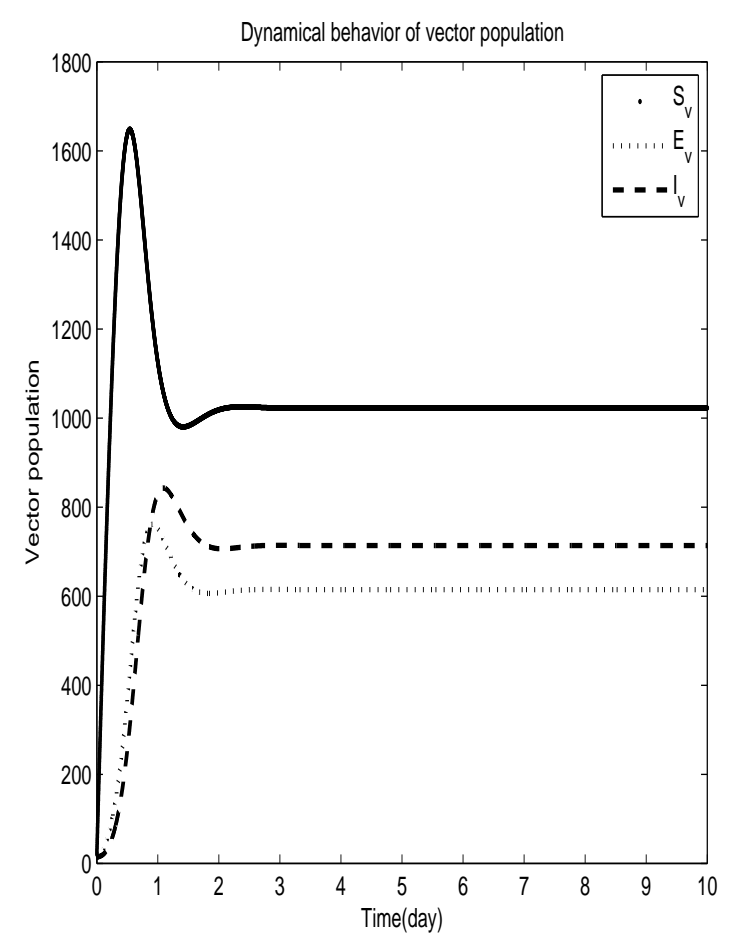

Fig. 4: The plot represents the vector population.

human and vector class, a transmission coefficient between Vectors of human exposed and infected vector.

\section{Appendix 1}

$J_{0}=\left[\begin{array}{ccccccc}-\mu_{0}-\alpha_{1} & 0 & 0 & \lambda_{h} & 0 & 0 & -\beta_{1}\left(\frac{a_{1}}{\mu_{0}+\alpha_{1}}\right) \\ -\alpha_{1} & -T_{1} & 0 & 0 & 0 & 0 & \beta_{1}\left(\frac{a_{1}}{\mu_{0}+\alpha_{1}}\right) \\ 0 & \alpha_{h} & -T_{2} & 0 & 0 & 0 & 0 \\ 0 & 0 & \delta_{h} & -T_{3} & 0 & 0 & 0 \\ 0 & 0 & -\beta_{2}\left(\frac{a_{2}}{\delta_{0}}\right) & 0 & -\delta_{0} & 0 & 0 \\ 0 & 0 & \beta_{2}\left(\frac{a_{2}}{\delta_{0}}\right) & 0 & 0 & -T_{4} & 0 \\ 0 & 0 & 0 & 0 & 0 & \alpha_{v} & -T_{5}\end{array}\right]$

By elementary row operation we get the characteristics equation of the above matrix is,

$$
\begin{aligned}
& \left(-M_{1}-\lambda\right)\left(-M_{1} T_{1}-\lambda\right)\left(-M_{1} T_{1} T_{2} \alpha_{1} \alpha_{h} \lambda_{h}-\lambda\right) \\
& \quad\left(M_{3}-\lambda\right)\left(-\delta_{0}-\lambda\right)\left(M_{3} \delta_{h} T_{4}-\lambda\right)\left(M_{4}-\lambda\right)=0
\end{aligned}
$$

where

$M_{1}=\mu_{0}+\alpha_{1}, M_{2}=\delta_{h} \alpha_{h} \mu_{0} \beta_{1} S_{h}^{0}$, 
$M_{3}=-M_{1} T_{1} T_{2} T_{3}+\delta_{h} \alpha_{1} \alpha_{h} \lambda_{h}$,

$M_{4}=-M_{3} \delta_{h} T_{4} T_{5}+\alpha_{v} M_{2} T_{3} \beta_{2} S_{v}^{0} \alpha_{v}$

$\lambda_{1}=-M_{1}<0, \lambda_{2}=-M_{1} T_{1}<0$,

$\lambda_{3}=-M_{1} T_{1} T_{2} \alpha_{1} \alpha_{h} \lambda_{h}<0, \lambda_{4}=-\delta_{0}<0$,

$\lambda_{5}=M_{3}, \lambda_{6}=M_{3} \delta_{h} T_{4}, \lambda_{7}=M_{4}$,

$\lambda_{5}<0 \Leftrightarrow M_{3}<0, M_{3}<0$,

putting the value of $M_{3}$ and $M_{1}$ we obtain,

$M_{1} T_{1} T_{2} T_{3}-\delta_{h} \alpha_{1} \alpha_{h} \lambda_{h}>0$.

and the condition,

$\left(\mu_{o}+\alpha_{1}\right)>\frac{\delta_{h} \alpha_{1} \alpha_{h} \lambda_{h}}{T_{1} T_{2} T_{3}}$.

$$
\lambda_{6}<0 \Leftrightarrow M_{3} \delta_{h} T_{4}<0, \quad M_{3} \delta_{h} T_{4}<0,
$$

putting the value of $M_{3}$ we get, $\quad\left(\mu_{o}+\alpha_{1}\right)>\frac{\delta_{h} \alpha_{1} \alpha_{h} \lambda_{h}}{T_{1} T_{2} T_{3}}$. $\lambda_{7}<0 \Leftrightarrow M_{4}<0, \quad M_{4}<0$, using the value of $M_{4}$ $M_{3} \delta_{h} T_{4} T_{5}-\alpha_{v} M_{2} T_{3} \beta_{2} S_{v}^{0} \alpha_{v}>0$, using $M_{3}$ and $M_{2}$ and after the arrangement we get,

$$
\begin{gathered}
\left(\mu_{o}+\alpha_{1}\right) T_{1} T_{2} T_{3} \delta_{h} T_{4} T_{5}+\alpha_{v}^{2} \delta_{h} \alpha_{h} \mu_{0} \beta_{1} \frac{a_{1}}{\left(\mu_{0}+\alpha_{1}\right)} T_{3} \beta_{2}\left(\frac{a_{2}}{\delta_{0}}\right) \\
+\delta_{h}^{2} \alpha_{h} \lambda_{h} T_{3} \mu_{0} T_{4} T_{5} \delta_{0}\left(1-R_{0}\right)+\delta_{h}^{2} \alpha_{h} \lambda_{h} T_{3} R_{0}\left(\delta_{0} \frac{\left(\mu_{0}+\alpha_{1}\right)}{\mu_{0} T_{4} T_{5}}\right)>0 .
\end{gathered}
$$

So the eigenvalues corresponds to the above jacobian matrix have negative real parts if $\delta_{0}>\frac{\left(\mu_{0}+\alpha_{1}\right)}{\mu_{0} T_{4} T_{5}}$ and $\left(\mu_{o}+\alpha_{1}\right)>\frac{\delta_{h} \alpha_{1} \alpha_{h} \lambda_{h}}{T_{1} T_{2} T_{3}}$.

Thus the DFE around $E_{1}$ of the system (1) is stable locally asymptotically.

\section{Appendix 2}

$J_{*}=\left[\begin{array}{cccccc}-\mu_{0}-\beta_{1} I_{v}^{*}-\alpha_{1} & 0 & 0 & 0 & 0 & -\beta_{1} S_{h}^{*} \\ \beta_{1} I_{v}^{*}+\alpha_{1} & -T_{1} & 0 & 0 & 0 & \beta_{1} S_{h}^{*} \\ 0 & \alpha_{h} & -T_{2} & 0 & 0 & 0 \\ 0 & 0 & -\beta_{2} S_{v}^{*}-\delta_{0}-\beta_{2} I_{h}^{*} & 0 & 0 \\ 0 & 0 & \beta_{2} S_{v}^{*} & \beta_{2} I_{h}^{*} & -T_{4} & 0 \\ 0 & 0 & 0 & 0 & \alpha_{v} & -T_{5}\end{array}\right]$.

Using elementary row operation we get the characteristics equation for the above matrix is,

$$
\begin{array}{r}
\left(-M_{1}-\lambda\right)\left(-M_{1} T_{1}-\lambda\right)\left(-M_{1} T_{1} T_{2}-\lambda\right)\left(-\delta_{0}-\lambda\right) \\
\left(-\delta_{0} M_{1} T_{1} T_{2} T_{4}-\lambda\right)\left(M_{4}-\lambda\right)=0,
\end{array}
$$

where,

$$
\begin{aligned}
M_{1}= & \mu_{0}+\beta_{1} I_{v}^{*}+\alpha_{1}, \\
M_{2}= & \beta_{1} I_{v}^{*}+\alpha_{1}, \\
M_{3}= & \delta_{0} \beta_{2} S_{v}^{*} \alpha_{h} \mu_{0} \beta_{1} S_{h}^{*}, \\
M_{4}= & -\delta_{0} M_{1} T_{1} T_{2} T_{4} T_{5}+\alpha_{v} \delta_{0} \beta_{2} \S_{v}^{*} \alpha_{h} \mu_{0} \beta_{1} S_{h}^{*} . \\
& \lambda_{1}=-M_{1}<0, \\
& \lambda_{2}=-M_{1} T_{1}<0, \\
& \lambda_{3}=-M_{1} T_{1} T_{2} T_{4}<0, \\
& \lambda_{4}=-\delta_{0}<0, \\
& \lambda_{5}=-\delta_{0} M_{1} T_{1} T_{4} T_{2}<0, \\
\lambda_{6}= & M_{4} .
\end{aligned}
$$

$\lambda_{6}<0 \Leftrightarrow M_{4}<0, \quad M_{4}<0$, putting the value of $S_{v}^{*}$, $S_{h}^{*}$ and $M_{1}, \delta_{0} M_{1} T_{1} T_{2} T_{4} T_{5}-\alpha_{v} \delta_{0} \beta_{2} S_{v}^{*} \alpha_{h} \mu_{0} \beta_{1} S_{h}^{*}>0$, after the simplifications and taking some arrangements we get,

$$
\begin{gathered}
{\left[\delta_{0} \mu_{0} T_{4} T_{5} \beta_{2} a_{1}+\delta_{0} a_{1} T_{4} T_{5} \beta_{2} a_{1}^{2}\right] I_{h}^{* 2}+\left[\alpha_{v} \delta_{0} \beta_{2} \alpha_{h} \mu_{0} \beta_{1} a_{2} \delta_{0}\left(R_{0}-1\right)\right.} \\
+\delta_{0} a_{2} \beta_{1} \beta_{2}\left(\left(\mu_{0}+\alpha_{1}\right) T_{1} T_{2} T_{4} T_{5}-\alpha_{v} \delta_{v} \alpha_{h} \mu_{0}\right) R_{0} \\
\left.\left.+\beta_{2} \beta_{1} \alpha_{h} \alpha_{v} a_{2}\left(\delta_{0}^{2}\left(\mu_{0}+\alpha_{1}\right)-a_{1} a_{2} \beta_{1} \beta_{2}\right)+2 \delta_{0}^{2}\left(\mu_{0}+\alpha_{1}\right) \beta_{2} a_{1} T_{4} T_{5}\right)\right] I_{h}^{*} \\
+\delta_{0}^{3}\left(\mu_{0}+\alpha_{1}\right) T_{4} T_{5} a_{1}+\delta_{0}^{2} \beta_{1} a_{2} T_{4} T_{5} a_{1} \alpha_{h}>0 .
\end{gathered}
$$

So the eigenvalues belongs to above jacobian matrix have negative real parts if $R_{0} \geq 1$, $\left(\mu_{0}+\alpha_{1}\right) T_{1} T_{2} T_{4} T_{5}>\alpha_{v} \alpha_{h} \mu_{0} \delta_{0} \quad$ and $\delta_{0}^{2}\left(\mu_{0}+\alpha_{1}\right) \geq a_{1} a_{2} \beta_{1} \beta_{2}$. So the endemic equilibrium point $E_{2}$ of the system (1) is locally asymptotically stable. This completes the proof of the theorem.

\section{Acknowledgement}

The authors are grateful to the anonymous reviewers for careful reading of the original manuscripts that greatly improved the presentation of this work.

\section{References}

[1] K. Hattaf, M. Rachik, S. Saadi, N. Yousfi, Optimal control of treatment in Basic virus infection model, Appl. Math. Sci., 3, 949-958 (2009).

[2] G. Cruz-Pacheco, L. Esteva, Modelling the dynamics of west nile virus, Bull. Math. Biol., 67, 1157-1172 (2005).

[3] G. Zaman, Y. H. Kang, I. H. Jung, Stability analysis and optimal vaccination of an SIR epidemic model, BioSystems, 93, 240-249 (2008).

[4] G. Zaman, Y. H. Kang, I. H. Jung, Optimal vaccination and treatment in the SIR epidemic model, Proc. KSIAM, 3, 31-33 (2007). 
[5] G. Zaman, Qualitative behavior of giving up smoking model, Bull. Mal. Math. Sci. Soc., acceptedpapers/2009-07-023-R1.

[6] N. Chitnis, T. Smith, R. Steketee, A mathematical model for the dynamics of malaria in mosquitoes feeding on a heterogeneous host population, J. Biol. Dyn., 2259-285 (2008).

[7] M. Derouich, A. Boutayeb, Mathematical modelling and computer simulations of Dengue fever, App. Math. Comput., 177, 528-544 (2006).

[8] L. Esteva, C. Vergas, A model for dengue disease with variable human populations, J. Math. Biol., 38, 220-240 (1999).

[9] P. Pongsuumpun, T. Miami, R. Kongnuy, Age structural transmission model for leptospirosis, The third International symposium on Biomedical engineering, 411-416 (2008).

[10] W. Triampo, D. Baowan, I. M. Tang, N. Nuttavut, J. WongEkkabut, G. Doungchawee, A simple deterministic model for the spread of leptospirosis in Thailand, Int. J. Bio. Med. Sci., 2, 22-26 (2007).

[11] G. Zaman, Dynamical behavior of leptospirosis disease and role of optimal control theory, Int. J. Math. Comp., 7.j10. (2010).

[12] G. Zaman, M. A. khan, S. Islam, Modeling dynamical interaction between leptospirosis infected vector and human population, Appl. Math. Sci, 6, 1287-1302 (2012).

[13] J. P. LaSalle, The Stability of Dynamical systems, SIAM, Philadelphia, PA, (1976).

[14] V. Lakshmikantham, S. leela, A. A. Martynyuk, Stability analysis of non linear systems, Marcel. Dikker. Inc, NewYork, Basel, (1989).

[15] K. P. Hadeler, P. van den Driessche, Backward bifurcation in epidemic model, Math. Biosci., 146, 15-35 (1997).

[16] O. Sharomi, C. N. Podder, A. B. Gumel, E. H. Elbasha, J.watmough, Role of incident function in vaccine-induced backward bifurcation in some HIV models, Math. Biosci., 210, 436-463 (2007).

[17] P. Van den Driessche, J. Watmough, Reproduction number and sub-threshold endemic equilibria for compartmental models of disease transmission,Math. Biosci., 180, 29-48 (2002).

[18] ZEB, A., Zaman, G., MOMANI, S., ERTRK, V. S., Solution of an SEIR Epidemic Model in Fractional Order. VFAST Transactions on Mathematics, 1, (2013).

[19] Ullah, R., Zaman, G., Islam, S., Stability analysis of a general SIR epidemic model. VFAST Transactions on Mathematics, 1, (2013).

[20] Saddiq, S. F., Khan, M. A., Khan, S. A., Ahmad, F., Ullah, M., Analytical solution of an SEIV epidemic model by Homotopy Perturbation method. VFAST Transactions on Mathematics, 1, (2013).

[21] Muhammad Altaf Khan, Saeed Islam, Muhammad Arif, and Zahoor ul Haq, Transmission Model of Hepatitis B Virus with the Migration Effect, BioMed Research International, 2013, 10 pages (2013).

[22] Muhammad Altaf Khan, Saeed Islam, Sher Afzal Khan, and Gul Zaman, Global Stability of Vector-Host Disease with Variable Population Size, BioMed Research International, 2013, 9 pages (2013).

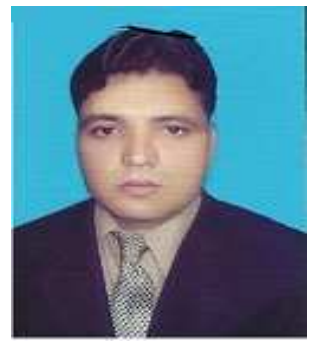

Muhammad Altaf Khan a $\mathrm{PhD}$ Scholar in Department of Mathematics Abdul Wali Khan University Mardan, Pakistan. His research interests are in the areas of applied mathematics, Mathematical Biology, Fractional order and mathematical physics. He has published more than 10 research articles in reputed international journals of mathematical and Medical sciences. He is referee for the British journal of Mathematics and Computer Sciences.

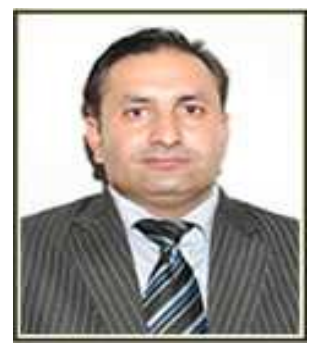

Saeed Islam He works as Associate Professor and Chairman, the Department of Mathematics, Abdul Wali Khan University Mardan, Pakistan. His research interest is the modeling and simulation using linear and nonlinear differential equations. He has more than 150 research articles in reputed journals listed in JCR and Thomson Master List. He supervised $5 \mathrm{PhDs}$ in COMSATS, Islamabad and Islamia College University Peshawar. He has been supervising more than 15 students of MPhil and PhDs. For his valuable research contribution, the government of Pakistan awarded him the Quaid-i-Azam Gold medal.

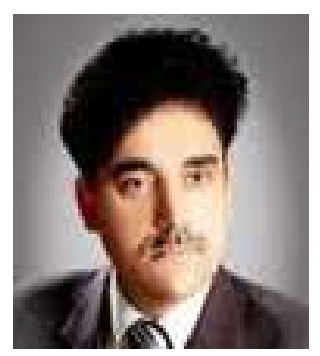

Sher Afzal Khan He works as Associate Professor and Chairman, the Department of Computer Sciences, Abdul Wali Khan University Mardan. His research interest is the modeling and simulation using differential equations, fuzzy logic, Petri net and $\mathrm{Z}$. He has many research articles in reputed journals listed in JCR. He is supervising 6 students of MPhil and PhDs. He is the chairman of the Virtual Foundation for Advancement of Science and Technology (http://vfast.org/), editor in chief of VAWKUM transactions on Computer Sciences (http://vfast.org/index.php/VTCS). Currently, he is the conference chair of the 'International Conference on Computational and Social Sciences (ICCSS-2013)' (http://iccss13.vfast.org/). 